\title{
Submillimeter observing conditions on Cerro Chajnantor
}

Simon J. E. Radford, Riccardo Giovanelli, George E. Gull, Charles P. Henderson

Simon J. E. Radford, Riccardo Giovanelli, George E. Gull, Charles P. Henderson, "Submillimeter observing conditions on Cerro Chajnantor," Proc. SPIE 7012, Ground-based and Airborne Telescopes II, $70121 Z$ (17 July 2008); doi: 10.1117/12.789335

Event: SPIE Astronomical Telescopes + Instrumentation, 2008, Marseille, France 


\title{
Submillimeter observing conditions on Cerro Chajnantor
}

\author{
Simon J. E. Radford, ${ }^{\mathrm{a}^{*}}$ Riccardo Giovanelli, ${ }^{\mathrm{b}}$ \\ George E. Gull, ${ }^{\mathrm{b}}$ and Charles P. Henderson ${ }^{\mathrm{b}}$ \\ ${ }^{a}$ California Institute of Technology, Pasadena, CA 91125, USA \\ ${ }^{\mathrm{b}}$ Cornell University, Ithaca, NY 14853, USA
}

\begin{abstract}
Consistently superb observing conditions are crucial for achieving the scientific objectives of a ground based telescope. For observations at submillimeter wavelengths, choosing a site with very little atmospheric water vapor is paramount. In northern Chile, the high Andes near San Pedro de Atacama are among the highest and driest places on Earth. At the $5000 \mathrm{~m}$ Chajnantor plateau, long term measurements have demonstrated observing conditions are excellent for submillimeter astronomy. Even better conditions prevail on higher mountain peaks in the vicinity. For the CCAT, we have selected a candidate site at $5612 \mathrm{~m}$ near the summit of Cerro Chajnantor. Radiosonde measurements, meteorological data, and measurements of the $350 \mu \mathrm{m}$ transparency all indicate submillimeter observing conditions are consistently better at the CCAT site than at the plateau.
\end{abstract}

Keywords: atmosphere, site testing, radio telescope

\section{INTRODUCTION}

To realize the full scientific potential of a ground based telescope, consistently superb observing conditions are crucial. At submillimeter wavelengths, the atmosphere is only partially transparent because of pressure broadened molecular absorption lines. Because water vapor is the primary impediment to atmospheric transparency, the best observatory sites are very dry, typically on high altitude mountains. Long recognized as a great site, the $4100 \mathrm{~m}$ summit of Mauna Kea, Hawaii, rises above an inversion layer that traps marine moisture at lower altitudes. Mauna Kea is now the location of major facilities for submillimeter astronomy (CSO, JCMT, SMA). Because of the very low temperatures, the saturation water vapor pressure on the Antarctica plateau is extremely low. A number of successful experiments, including the SPT, have been deployed at the $2835 \mathrm{~m}$ South Pole. Exploratory measurements have been made at higher sites including domes C (3100 m) and A (4200 m). In northern Chile, the high Andes near San Pedro de Atacama are among the highest and driest places on Earth. At the $5000 \mathrm{~m}$ Chajnantor plateau, long term measurements have demonstrated observing conditions are excellent for submillimeter astronomy. ${ }^{1}$ Undeveloped ten years ago, the plateau is now the location of several major new facilities (CBI, ASTE, APEX, NANTEN2, ALMA). Even better conditions prevail on the high mountain peaks surrounding the Chajnantor plateau. At $5500 \mathrm{~m}$ on Cerro Sairecabur, the SAO RLT has carried out pioneering observations at $\mathrm{THz}$ frequencies. ${ }^{2}$ The CCAT is a proposed $25 \mathrm{~m}$ diameter telescope for submillimeter astronomy. ${ }^{3}$ For CCAT, we have selected a candidate site at $5612 \mathrm{~m}$ near the summit of Cerro Chajnantor. Three sets of evidence demonstrate submillimeter observing conditions on Cerro Chajnantor are better than on the Chajnantor plateau. Radiosonde measurements of the atmospheric structure and water vapor profile show the water vapor decreases with altitude. Simultaneous measurements of the ground level water vapor content show the higher site is drier. Finally, simultaneous measurements show the $350 \mu \mathrm{m}$ transparency is consistently better at the CCAT site than at the plateau.

\section{ATMOSPHERIC PROFILES}

Starting in 1998, Cornell, NRAO, ESO, SAO, and NAOJ conducted a series of radiosonde launches from the Chajnantor plateau. ${ }^{4}$ The balloon borne sensor packages measured pressure, temperature, and relative humidity, from which the water vapor content was calculated. Wind speed and direction were inferred from the tracking data. Although the schedule was irregular, launches were made both day and night at different times of year to sample any diurnal and seasonal variations.

\footnotetext{
*sradford@caltech.edu
} 
Taken all together, the radiosonde data show a simple atmospheric profile above the Chajnantor plateau (Fig. 1). For water vapor, the median exponential scale height of all launches is $1.1 \mathrm{~km}$. At night the water vapor is concentrated at lower altitudes; the median scale hight for night launches is only about $750 \mathrm{~m}$.

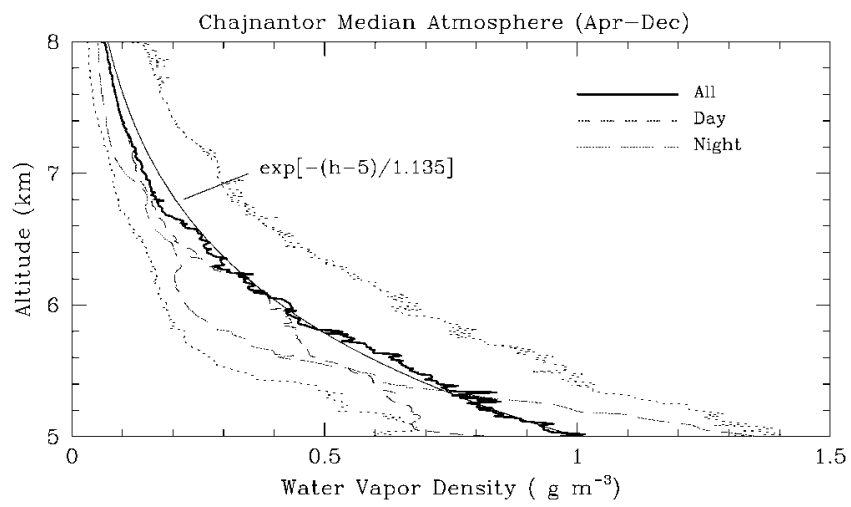

Fig. 1. Distribution of water vapor density profiles above the Chajnantor plateau. ${ }^{4}$ The median profile of 108 radiosonde launches (thick line) is fit by an exponential with a scale height of $1.1 \mathrm{~km}$ (thin line). The dotted lines show the first and third quartile profiles. The median profile of 30 night launches (long dash line) shows a shorter scale height than the median of 65 daytime launches (short dash line).

Individual launches can show marked deviations from the collective behavior. In particular, there is often evidence for a temperature inversion layer at moderate altitude above the plateau that traps water vapor below it. Especially at night, the inversion layer height can be quite low, below 5500 m (Fig. 2)

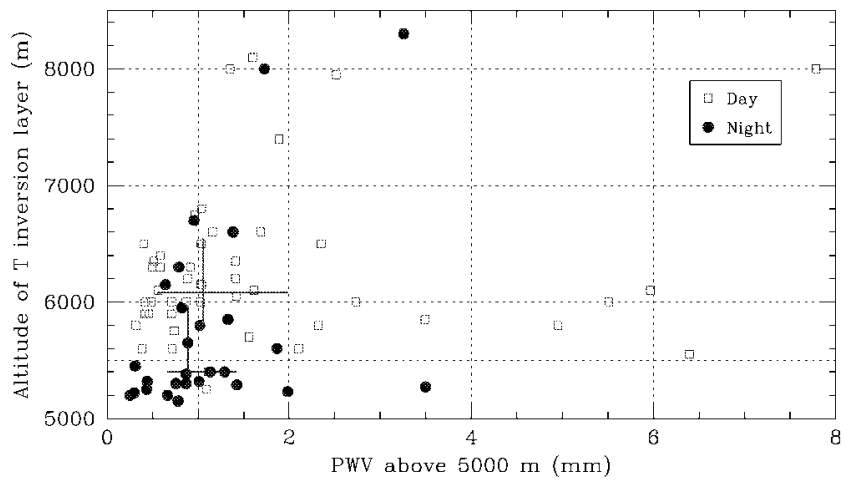

Fig. 2. Altitude of temperature inversion layers identified in daytime (open squares) and night (solid circles) radiosonde profiles and water vapor column density (PWV) above the Chajnantor plateau. ${ }^{4}$ The crosses show the median altitude and PWV values; the span of the crosses' arms extends from first to third quartiles.

\section{METEOROLOGY MEASUREMENTS}

Of the several peaks in the vicinity of the Chajnantor plateau, weather stations were deployed on two: Cerro Negro and Cerro Chajnantor. These instruments are Weatherpak 2000 models from Coastal Environmental Systems. West of the Chajnantor plateau, the land descends steadily for $2500 \mathrm{~m}$ over $30 \mathrm{~km}$ to the Salar de Atacama. Cerro Negro, $9 \mathrm{~km}$ west of the plateau, is the same altitude as the plateau but rises about $500 \mathrm{~m}$ above its surroundings. Cerro Negro provides an opportunity to investigate whether the water vapor density depends on local relief or absolute altitude. Cerro Chajnantor rises $600 \mathrm{~m}$ above the plateau $5 \mathrm{~km}$ to the NNE. The candidate CCAT site (Fig. 3) is about $200 \mathrm{~m}$ east of and $25 \mathrm{~m}$ below the summit. Cerro Chajnantor is same altitude as the SAO RLT ${ }^{2}$ on Cerro Sairecabur $32 \mathrm{~km}$ to the NNW. For comparison, used the weather data recorded by the Cosmic Background Imager at the Caltech Chajnantor Observatory on the plateau. To avoid complications when one or another station was offline, comparisons were made only between measurements recorded within 15 min of each other. This allows simple comparison of cumulative distributions. 
Table 1. Weather station locations. Coordinates refer to the WGS84 datum.

\begin{tabular}{|c|c|c|c|c|c|c|}
\hline Location & code & S latitude & W longitude & altitude $[\mathrm{m}]$ & start & end \\
\hline Cerro Chajnantor & $\mathrm{cc}$ & $22^{\circ} 59^{\prime} \quad 8^{\prime \prime}$ & $66^{\circ} 44^{\prime} 25^{\prime \prime}$ & 5612 & 2006 May & 2008 Jun \\
\hline Cerro Negro & ng & $23^{\circ} 1^{\prime} 15^{\prime \prime}$ & $67^{\circ} 51^{\prime} \quad 3 "$ & 5000 & 2004 Dec & 2007 Nov \\
\hline Chajnantor plateau & $\mathrm{cb}$ & $23^{\circ} 1^{\prime} 43^{\prime \prime}$ & $67^{\circ} 45^{\prime} 42^{\prime \prime}$ & 5050 & 1999 Nov & 2008 Jun \\
\hline
\end{tabular}

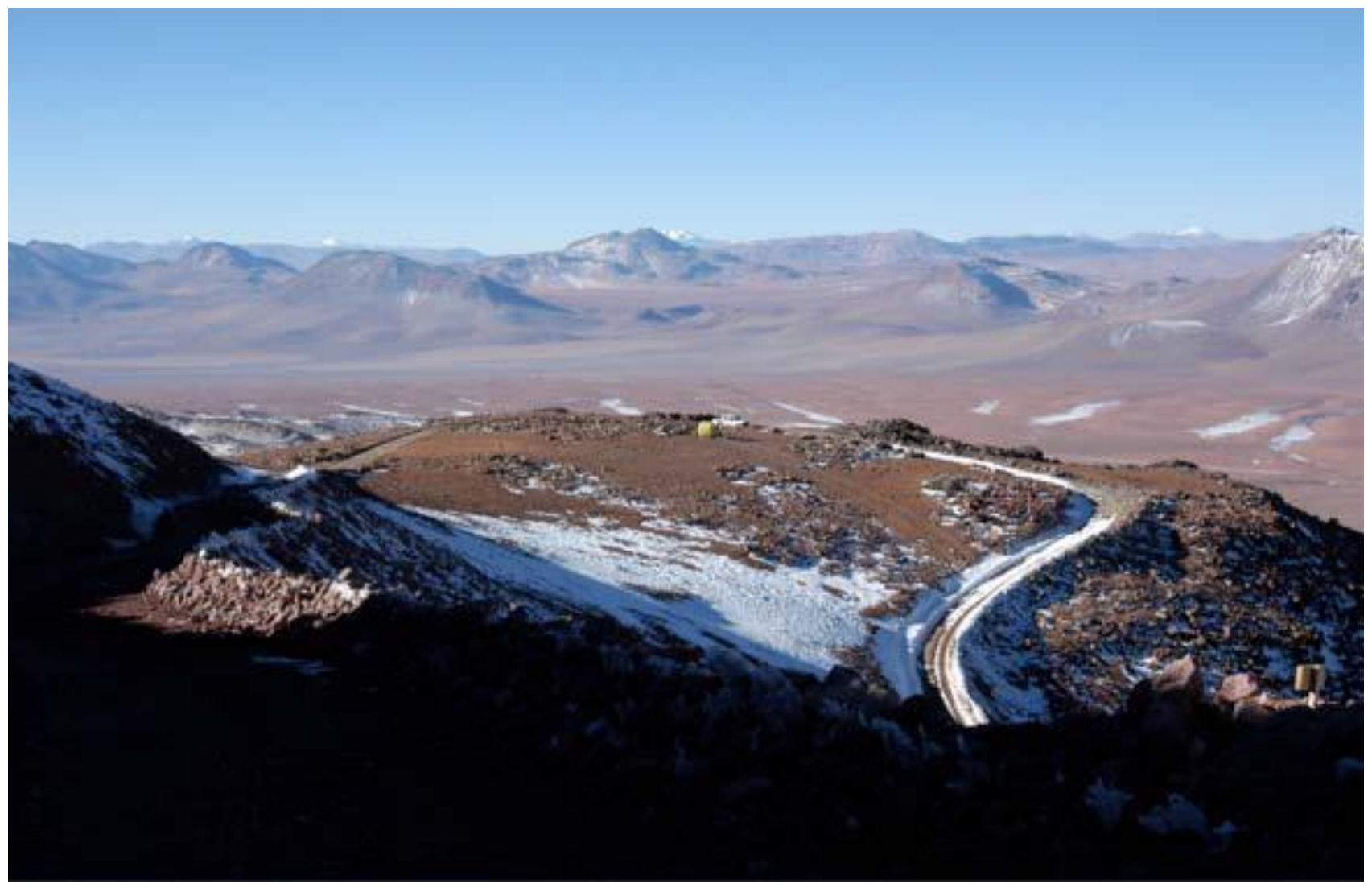

Fig. 3. Weather station at candidate CCAT site near the summit of Cerro Chajnantor. 
Cerro Chajnantor is about $3{ }^{\circ} \mathrm{C}$ colder than the plateau (Fig. 4), consistent with the altitude difference and the lapse rate of the standard atmosphere, $6.5^{\circ} \mathrm{C} \mathrm{km}^{-1}$. Cerro Negro is a bit warmer than the plateau.

At all three locations, the wind blows predominantly from the west. The windspeed on both Cerro Chajnantor and Cerro Negro is lower than at the Chajnantor plateau (Fig. 5). On Cerro Chajnantor, the CCAT weather station is likely sheltered by the peak of the mountain $200 \mathrm{~m}$ to the east. In addition, the Chajnantor plateau is westward of a pass between Cerro Chajnantor and Cerro Chascón that funnels the prevailing westerly wind.
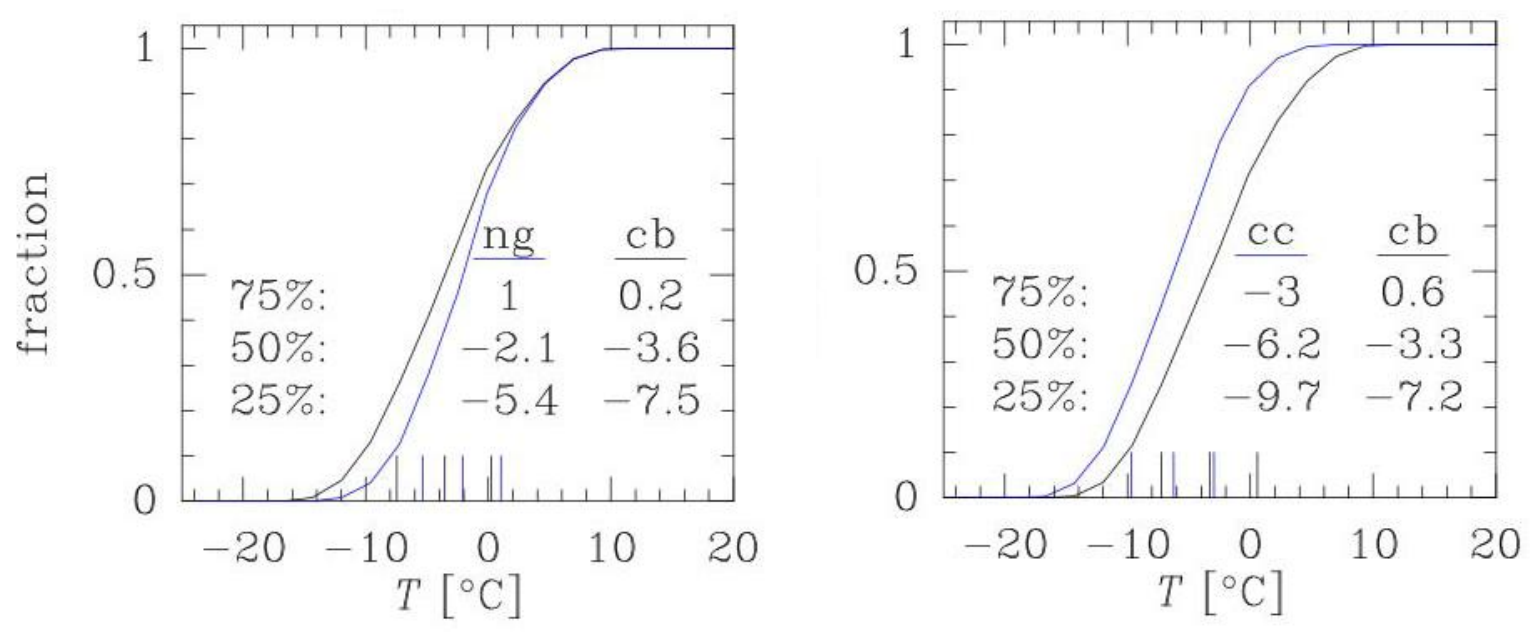

Fig. 4. Cumulative distributions of air temperature at Cerro Chajnantor (cc), Cerro Negro (ng), and the Chajnantor plateau (cb). Because these are comparisons of simultaneous data collected over different periods, there are small differences in the reference $(\mathrm{cb})$ distributions.
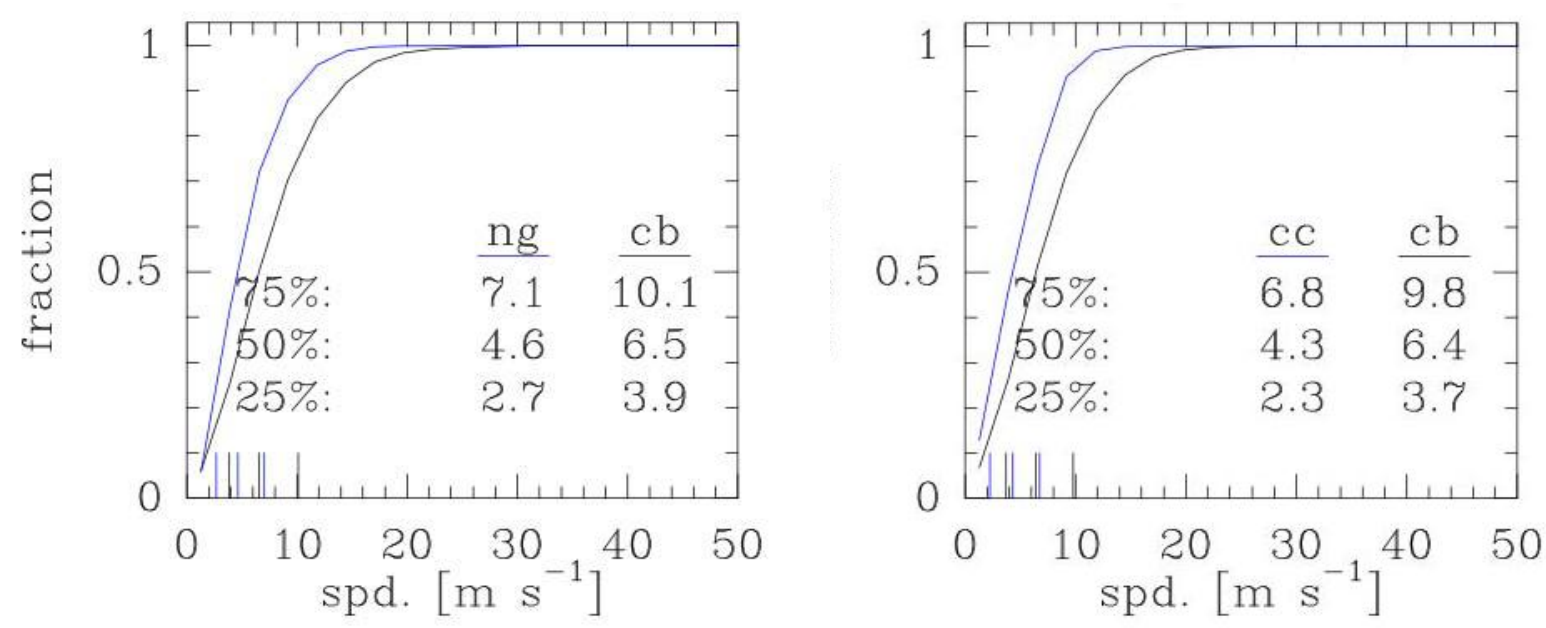

Fig. 5. Cumulative distributions of wind speed at Cerro Chajnantor (cc), Cerro Negro (ng), and the Chajnantor plateau (cb). Because these are comparisons of simultaneous data collected over different periods, there are small differences in the reference $(\mathrm{cb})$ distributions. 


\section{GROUND LEVEL WATER VAPOR DENSITY}

Although the ground level humidity is, at best, an imperfect measure of the water vapor content of the entire atmospheric column, simultaneous measurements at nearby sites may be a valid indicator of differences between them, especially if the atmospheric profile is simple. The water vapor density is calculated from the measured values of the temperature, pressure, and relative humidity.,

On Cerro Negro and at the Chajnantor plateau the distributions of water vapor density are almost identical (Fig. 6) and the measurements are strongly correlated. These data suggest the water vapor content of the atmospheric column is similar in these locations. Apparently the local relief of Cerro Negro above its surroundings has little effect on the water vapor density. The data suggest water vapor is stratified and depends on absolute altitude.

On Cerro Chajnantor, on the other hand, the water vapor density is noticeably lower than at the Chajnantor plateau (or on Cerro Negro). The median water vapor density on Cerro Chajnantor is $76 \%$ of that at the plateau. The measurements are also strongly correlated, as expected for two nearby sites sharing the same weather patterns. In this case, the correlation diagram shows a marked asymmetry: there are a significant number of measurements, falling in the lower right corner of the diagram, where the water vapor density on Cerro Chajnantor is much less than at the plateau. Such instances are not seen in the Cerro Negro data. This is, however, the signature expected if a temperature inversion trapped water vapor below the altitude of Cerro Chajnantor.
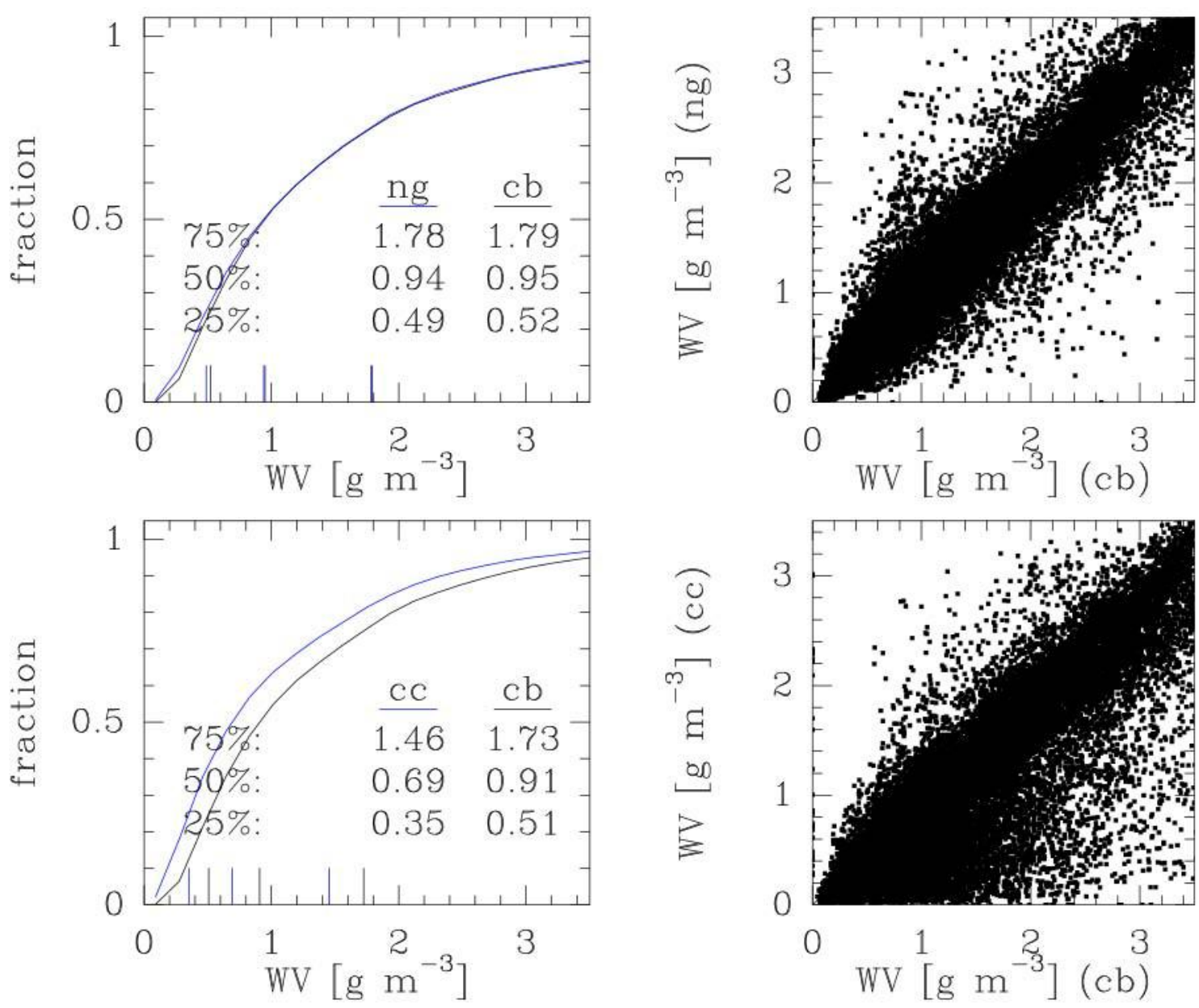

Fig. 6. Cumulative distributions and correlations of ground level water vapor density at Cerro Chajnantor (cc), Cerro Negro (ng), and the Chajnantor plateau (cb). Because these are comparisons of simultaneous data collected over different periods, there are small differences in the reference $(\mathrm{cb})$ distributions. 


\section{SUBMILLIMETER TRANSPARENCY}

Direct measurements of the atmospheric transparency at $350 \mu \mathrm{m}$ wavelength were made with two tipping radiometers, one on Cerro Chajnantor and the other at the Caltech Chajnantor Observatory on the plateau. Prior to deployment on Cerro Chajnantor, the two radiometers were operated side by side on the plateau (Fig. 7). The side by side measurements demonstrate the data from the radiometers can be compared directly.

On Cerro Chajnantor, the measured $350 \mu \mathrm{m}$ transparency is consistently better than at the plateau (Fig. 8). The data are strongly correlated. Regression of the correlation indicates the radiometers have an instrumental offset in the apparent $350 \mu \mathrm{m}$ optical depth of about $\tau_{0} \approx 0.7$ and $\tau(\mathrm{cc})-\tau_{0}=0.6\left[\tau(\mathrm{cb})-\tau_{0}\right]$. These data indicate submillimeter observing conditions on Cerro Chajnantor are significantly better than on the Chajnantor plateau.
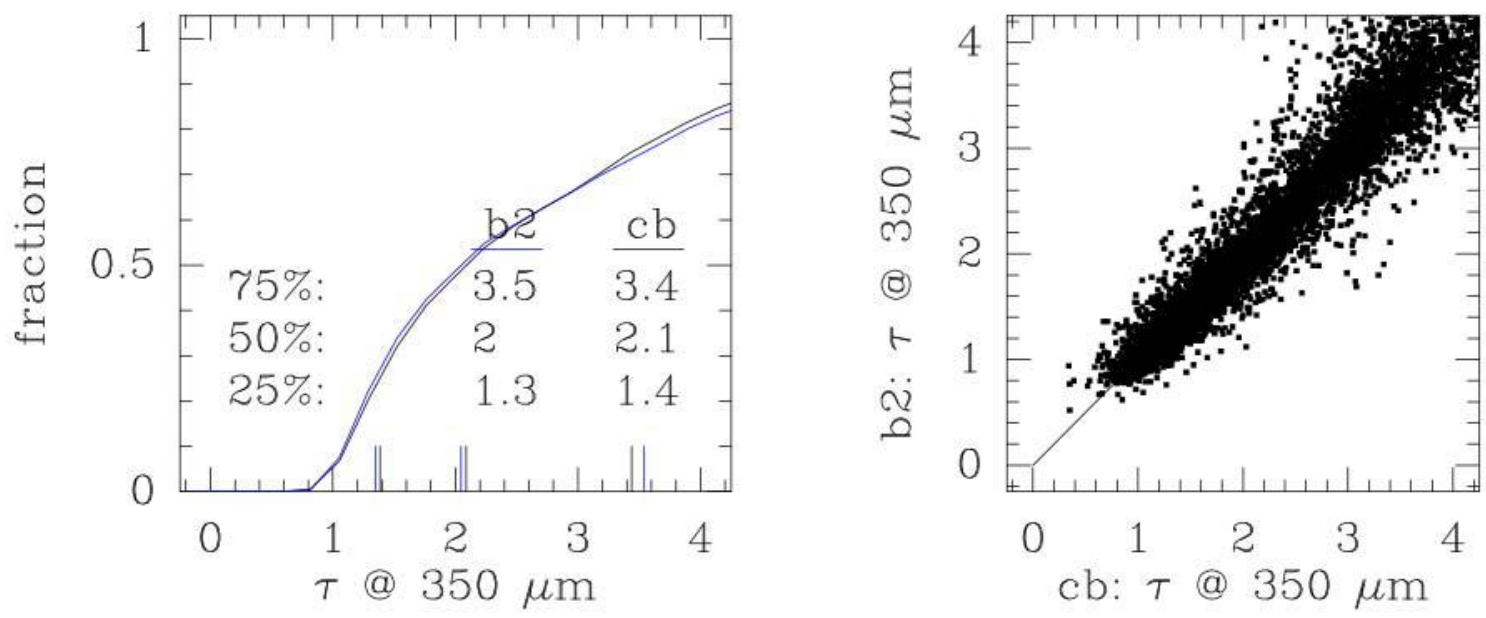

Fig. 7. Cumulative distributions and correlation of $350 \mu \mathrm{m}$ optical depth $(\tau)$ measured simultaneously from 2005 May to 2006 May with two tipping radiometers side by side the Chajnantor plateau.
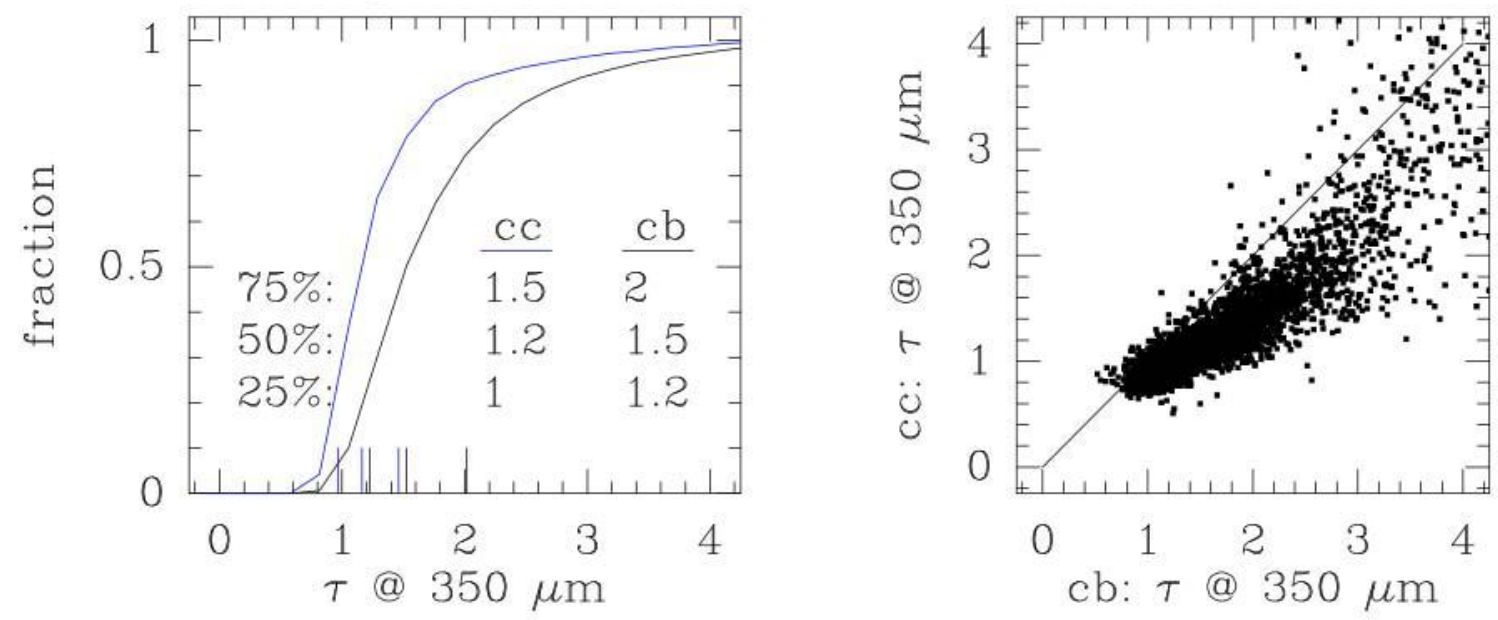

Fig. 8. Cumulative distributions and correlation of $350 \mu \mathrm{m}$ optical depth $(\tau)$ measured simultaneously from 2005 May to 2006 November with two tipping radiometers, one on Cerro Chajnantor (cc) and the other at the Chajnantor plateau (cb). 


\section{SUMMARY}

In northern Chile, sites in the high Andes near San Pedro de Atacama enjoy excellent conditions for submillimeter astronomy. Even though the $5000 \mathrm{~m}$ Chajnantor plateau is already one of the best sites known, the higher peaks in the vicinity enjoy even better conditions. For the CCAT, we have selected a candidate site at $5612 \mathrm{~m}$ near the summit of Cerro Chajnantor. Three sets of evidence demonstrate submillimeter observing conditions on Cerro Chajnantor are better than on the Chajnantor plateau. Radiosonde measurements of the atmospheric profile above the plateau show the median exponential scale height of water vapor is $1.1 \mathrm{~km}$, making Cerro Chajnantor drier than the plateau. This advantage over the plateau is enhanced when temperature inversions trap water vapor below the level of its summit. Simultaneous meteorological measurements show the ground level water vapor density is lower on Cerro Chajnantor than at the plateau. These data show a signature of temperature inversions as well. Simultaneous measurements with $350 \mu \mathrm{m}$ tipping radiometers indicate the submillimeter transparency is consistently better on Cerro Chajnantor than at the Chajnantor plateau.

\section{ACKNOWLEDGEMENTS}

We thank our friends who have contributed to these results, including C. Achermann, R. Bustos, N. Oyarce, R. Reeves, M. Uehara, A. Otárola, R. Blundell, and S. Paine. We thank Y. Yoshii and M. Doi for the access road on Cerro Chajnantor. The measurements were made with the agreement and support of the CONICYT and the U. Chile. This research was partially supported by the National Science Foundation.

\section{REFERENCES}

[1] Radford, S. J. E., and Holdaway, M. A., "Atmospheric Conditions at a Site for Submillimeter Wavelength Astronomy," in Advanced Technology MMW, Radio, and Terahertz Telescopes, ed. T. G. Phillips, Proc. SPIE 3357, 486 (1998).

[2] Marrone, D. P., Battat, J., Bensch, F., Blundell, R., Diaz, M., Gibson, H., Hunter, T., Meledin, D., Paine, S., Papa, D. C., Radford, S. J. E., Smith, M., and Tong, E., “A Map of OMC-1 in CO J = 9 - 8," Ap. J. 612, 940 (2004).

[3] Sebring, T. A., Radford, S., Giovanelli, R., Glenn, J., and Woody, D., "The Cornell Caltech Atacama Telescope Status and Technical Progress," Proc. SPIE 7020 (2008).

[4] Giovanelli, R., Darling, J., Henderson, C., Hoffman, W., Barry, D., Cordes, J., Eikenberry, S., Gull, G., Keller, L., Smith, J. D., and Stacey, G., "The Optical/Infrared Astronomical Quality of High Atacama Sites. II. Infrared Characteristics," PASP 113, 803 (2001).

[5] Buck, A. L., "New Equations for Computing Vapor Pressure and Enhancement Factor," J. App. Meteor. 20, 1527 (1981).

[6] Buck Research Instruments, "Model CR4 Hygrometer Operating Manual” (2005). 